\title{
An Easy to Use Augmented Reality Authoring Tool for Use in Examination Purpose
}

\author{
Ming-Jen Wang, Chien-Hao Tseng, and Cherng-Yeu Shen \\ No. 7, R\&D Rd. VI Hsinchu Science Park, Hsinchu, Taiwan (R.O.C.) \\ \{renny, c00how0 0 , c00cys 00$\}$ anchc.org. tw
}

\begin{abstract}
This paper introduces an augmented reality authoring tool that allows users to edit and publish an examination application with the Augmented Reality (AR) interface. The AR authoring system in this paper is constructed with the help of ARToolKit, a widely used open source for AR. This unique system can help users with no programming knowledge to build AR applications quickly and efficiently. 2D text, 3D content editing, and finger-based interaction are the three major components found in the AR authoring environment. AR content created by the user is stored in a separate txt file for easy sharing, modification, and reusability. A user testing of the published examination application was also conducted, and participants in the testing offered generally positive feedback.
\end{abstract}

Keywords: Augmented Reality, education, Human Computer Interaction.

\section{Background}

In order to introduce AR technology to the public and to content developers, many AR development tools have been proposed. These tools can generally be divided into two categories.

The first major category is AR-related library or software framework. Since the development of AR applications is technologically complex, using such systems as computer vision, computer graphics, image recognition, and so on, developing an AR application at a low-level programming environment often consumes considerable amounts of time. Therefore, in order to shorten AR development time, many AR related libraries and software frameworks have been proposed. Some of the most well known libraries and frameworks are ARToolKit [1], osgART [2], DWARF [3], MRT(Mixed Reality Toolkit) [4], ARTag [5], etc. Among these libraries, perhaps the most widely used is ARToolKit, a $\mathrm{C} / \mathrm{C}++$ based open source tracking library. The ARToolKit library is so popular that it has even been transferred into other computing languages, such as NyARToolKit (Java) and FLARToolKit (Flash ActionScript). Other than tracking issue, some libraries, such as OSGART, focuses on rendering issues related to AR. However, despite the many efforts expensed in the development of AR tools, most if not all of these tools are still only accessible and practically useful to professional programmers. The remainder of the universe of potential AR users are still waiting for tools which will provide them with a competitive amount of AR developing power. 
The second category is the GUI-based AR authoring tool. As opposed to textcommand based interfaces, GUI's provide more intuitive interaction for users. Before the GUI-based AR tool, a finished AR application might require programming and then de-bugging thousands of lines of code. Even with AR related open source libraries, developers still needed to invest significant time to learn how to use them. By introducing the GUI to the tool, developers now have a tool allowing them to complete AR applications on a point-and-click basis. AMIRE [6] is one of the GUI-based AR authoring tools. With it, users create AR applications without having to write any code. DART [7] is a plug-in for Adobe Director software which allows one to author AR content as well. ComposAR [8] provides another GUI-based tool for authoring AR content. Outside of the GUI solution, ComposAR provides an additional scripting interface for advanced users with computer programming experience who desire more control over the application design process. ATOMIC Authoring Tool [9] is among the examples of AR authoring interfaces made extremely simple.

We notice that most of the above mentioned tools only focus on building the relationship between AR Markers and 3D models. When the above tools are used to build complex AR applications, they either fail entirely or require further scripting to achieve the goal. For a non-programmer, AR application development is therefore highly restricted if only these tools are available. In this paper we propose a GUI based AR authoring tool that allows users to build AR applications with examination mechanisms and 3D model display functions free of any further coding. The user can answer pertinent questions at decision nodes by pointing to Yes or No Markers.

\section{System Design}

The system design has four key features:

First, we embed a Yes/No question examination mechanism in the AR authoring tool. From a pedagogical standpoint, examination is an important method in understanding the efficacy of a system of learning. However the examination process is often dry and not very attractive to students, especially younger ones. Also, a question might not be only text-based. It might be a graphical question that contains a $3 \mathrm{D}$ virtual model. Using a Marker-based AR application, users can interact with 3D models in a way that is very similar to how one interacts with real objects. Hence we hybridize the AR interface and examination to best take advantage of the most desirable traits of each system. To do so, a question and answer input interface is needed. In the authoring interface, a user can type in the question and choose the correct answer to the question in the question input section. If the question requires $3 \mathrm{D}$ virtual modeling in order to gain additional information, a user can also select a 3D model that has been previously made from the local computer to assist. If the 3D model's settings are not appropriate, the system also provides some basic functions to adjust the 3D model's size, translation and rotation parameters. Testing revealed that developing a 20 Yes/No question-based AR examination application using the system frequently required no more than ten minutes provided any $3 \mathrm{D}$ models and questions have been prepared beforehand.

Second, the reusability of the question data is considered. The system stores the question data in an external text file. All the questions and their answers, 3D models' 
saving paths, and Marker information are saved with predefined rules in a single txt file. There are two important purposes in doing so. First, saving the question data in an external file allows authors to exchange question data more easily via import/export protocols. This feature reduces the development time one step further when question data is readily obtainable. Second, the generation of questions is basically relying on the text file. Authors can readily understand most of the rules of the text file once they have examined it using the authoring system. This means the author can modify the questions in the text file directly without using the authoring system. This feature gives authors an easy and fast way to create or modify an AR examination application.

Third, the system is divided into two different modes, the authoring mode and the viewing mode, to provide the author and students the various functions they need. The system structure is shown in Fig. 1.

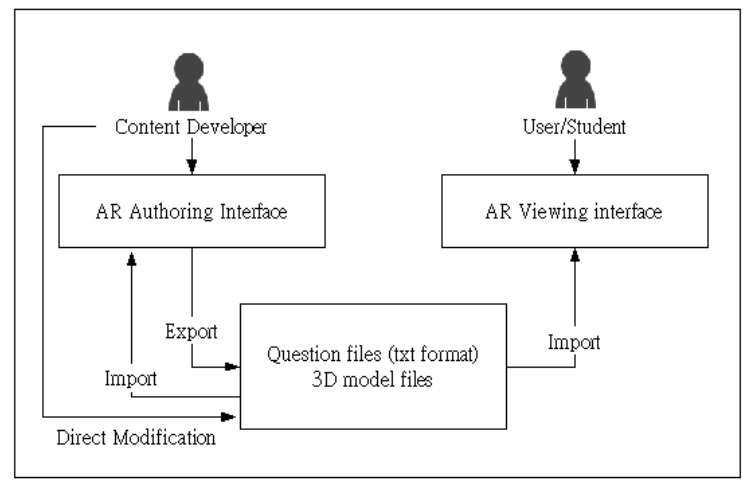

Fig. 1. The Authoring tool system structure of authoring and viewing modes

Fourth, we utilize a complete graphical user interface in the system. Target users of the system include teachers and students in elementary/high/senior high schools. Because the target users' knowledge of computer programming is assumed to be limited, a GUI-based interface will be more suitable for them. With our system, a few clicks and the input of some questions suffice for the author wishing to rapidly complete an application.

\section{Prior User Testing and Discussion}

We conducted a user testing to gather feedback and responses after using this system. The participants are all elementary students from the central region of Taiwan. There are a total of 24 students, all aged around 8 years old, who participated the experiment. To begin, we setup an AR examination application which consists of 10 questions using the authoring program. In the prior testing, we discovered that they weren't sure how long to "touch" the Yes/No marker to verify the choices of their answers. After being instructed with the time needed to press down on the marker in order to make the selection, all the students in this experiment were able to practice the interaction very 
quickly. Some of the students even finished all the 10 questions within 2 minutes. To conclude, 21 out of 24 students consider that the system is interesting and convenient to use and are willing to use the system as their future learning tool.

In this paper, we propose an AR developing tool that could assist content developers to create an interactive examination system with AR interface within a short amount of time. Comparing with the other AR authoring tools, the system is targeting at a specific purpose which is examination/education. For those tools that only support simple 3D models and Marker information linkage, the system we have developed could offer an easier and faster way for the content developers to create an examination with AR application. We also propose two different models for various users, content developers and as well as students. The data of the questions is also saved where it could be easily shared, exchanged or modified later on. In this user testing, we discover that most of the students offer positive feedback on the AR application built with the authoring tool. Nevertheless, the system still has room for further improvements. For one, the system could only support the Yes/No question-based examinations. If an author wants to develop a more complex examination or nonexamination project using the AR application, it cannot be done by using this system and that is one of the limitations of this system. In the future, we are going to modularize the system functions. With more AR interaction modules being developed, the program users would be given more freedom and choices in creating examinations with a simple authoring process.

\section{References}

1. Kato, H., Billinghurst, M.: Marker tracking and hmd calibration for a video-based augmented reality conferencing system. In: Proceedings of the 2nd IEEE and ACM International Workshop on Augmented Reality (1999)

2. Looser, J., Grasset, R., Seichter, H., Billinghurst, M.: OSGART - a pragmatic approach to MR. In: Industrial Workshop at ISMAR (2006)

3. Bauer, M., Bruegge, B., Klinker, G., MacWilliams, A., Reicher, T., Riss, S., Sandor, C., Wagner, M.: Design of a component-basedaugmented reality framework. In: Proceedings of the International Symposium on Augmented Reality (2001)

4. Freeman, R.: Mixed Reality Toolkit. MSc VIVE Final Year Project Report, Department of Computer Science, University College London (2004)

5. Fiala, M.: ARTag Revision 1, A Fiducial Marker System Using Digital Techniques NRC Technical Report (NRC 47419), National Research Council of Canada (2004)

6. Grimm, P., Haller, M., Paelke, V., Reinhold, S., Reimann, C., Zauner, J.: AMIRE - Authoring Mixed Reality. In: First IEEE International Augmented Reality Toolkit Workshop, Darmstadt,Germany (2002)

7. MacIntyre, B., Gandy, M., Dow, S., Bolter, J.D.: DART: A Toolkit for Rapid Design Exploration of Augmented Reality Experiences. In: Conference on User Interface Software and Technology (UIST 2004), Sante Fe, New Mexico (2004)

8. Seichter, H., Looser, J., Billinghurst, M.: ComposAR: An Intuitive Tool for Authoring AR Applications. In: Mixed and Augmented Reality, 7th IEEE/ACM International Symposium, Cambridge, UK (2008)

9. ATOMIC Authoring Tool, http://www.sologicolibre.org/projects/atomic/en/ 